\title{
A multi-dimensional approach to lichen sclerosus therapy
}

\author{
Luigi Laino ${ }^{1,2 *}$
}

${ }^{1}$ Dermatologic and Venereologic Centre Via Bixio, Rome, Italy

${ }^{2}$ Faculty at Dermatologic Institute for Research and Care - IRCCS, Rome Italy

Lichen sclerosus (LS) $[1,2,3]$ is an inflammatory dermatologic chronically relapsing disease with a potential for atrophy, hypertrophy, destructive scarring, functional impairment, leucoplachiae, and malignant evolution [4]. Therefore, early diagnosis, prompt treatment, and long-term follow-up of affected patients are mandatory. Spontaneous remissions are rare. Despite there isn't a causal therapy, LS can be controlled by adequate treatment: in several cases, it could be observed a complete remission of sign and symptoms. With early treatment, long-term sequelae such as destruction of anatomic structures and progression to squamous cell carcinoma may be prevented. In particular cases, could be very useful that a multidisciplinary approach, so is necessary that dermatologists, urologists and gynecologists have a solid knowledge of the disease and will not hesitate to cooperate if required. Since LS begins with uncharacteristic symptoms, a peculiar clinical examination, and if necessary, a histopathologic confirmation is required. Despite many efforts have been made, in recent years, to find an appropriate therapy that could cure effectively lichen sclerosus, there are many treatment failures. While many cases of LS must not be treated surgically, it is also true, that many LS are due to congenital or acquired alterations that caused the persistence of the disease; this anatomical changes can be identified as "trigger factors" for the auto-maintenance of LS signs and symptoms; in this occasion, only topical and/or micro-infiltrative approach could be insufficient for a long-term management and could delay or making inadequate the only topical and/or micro-infiltrative approaches. For this reason we have developed, over 6 year (2008 to present) in 58 patients ( 36 male, 22 female aged from 28 from 65 year-old suffering from moderate to severe lichen sclerosus, a multi-dimensional protocol that is based the following triad: conservative surgical therapy, topical therapy and micro-infiltrative therapy. We made a diagnosis of LS, both on the basis of clinical data and through biopsy, in compliance with the recent guidelines of the British Association of Dermatologists [5]. The patients were evaluated by the investigator on the Investigator's Global Assessment (IGA) and the Dermatology Life Quality Index (DLQI) [6].

We divided our patients into 2 groups, A $(=16$; male $=8$; female $=8)$ and $\mathrm{B}(=16 ;$ male $=8 ;$ female $=8)$, with homogeneous IGA and DLQI.

For group A patients, we proposed a multi-dimensional therapeutic approach that consisted in:

a) Conservative dermosurgical approach, limited to eliminate peculiar alteration as the presence of a short and sclerotic frenulum, partial or total sclerotic phimosis, balano-preputial/vulvar synechiae or leucoplastic areas, associated to histological exam.

b) Topical Therapy with clobetasolproprionate (CP) + Vit E emollient cream

c) Subdermal micro-infiltration with polydeoxyribonucleotide (PDRN)

Correspondence to: Dr. Luigi Laino, Director of Dermatologic and Venereologic Centre Via Bixio, Rome, Italy, Tel: +390645550661; Fax: +390690213462; E-mail: luigilaino@yahoo.it

Key words: Lichen sclerosus, phimosis, neoplastic vulvar condition

Received: March 10, 2015; Accepted: April 11, 2015; Published: April 13, 2015 
Table 1. Values median (range) of IGA and DLQI scores before and after therapy in group A and group B; comparisons are evaluated using paired Wilcoxon signed-rank for paired data.

\begin{tabular}{|l|c|c|c|c|c|}
\hline \multirow{2}{*}{ Variables } & \multicolumn{3}{|c|}{ Group A } & \multicolumn{2}{|c|}{ Group B } \\
\cline { 2 - 5 } & Beforetherapy & Aftertherapy & P value & Beforetherapy & $5(3-9)$ \\
\hline $\begin{array}{l}\text { IGA } \\
\text { Median (range) }\end{array}$ & $6(3-10)$ & $2(0-7)$ & 0.001 & 0.001 \\
\hline $\begin{array}{l}\text { DLQI } \\
\text { Median (range) }\end{array}$ & $15(8-19)$ & $7(3-11)$ & 0.001 & $14(8-19)$ \\
\hline
\end{tabular}

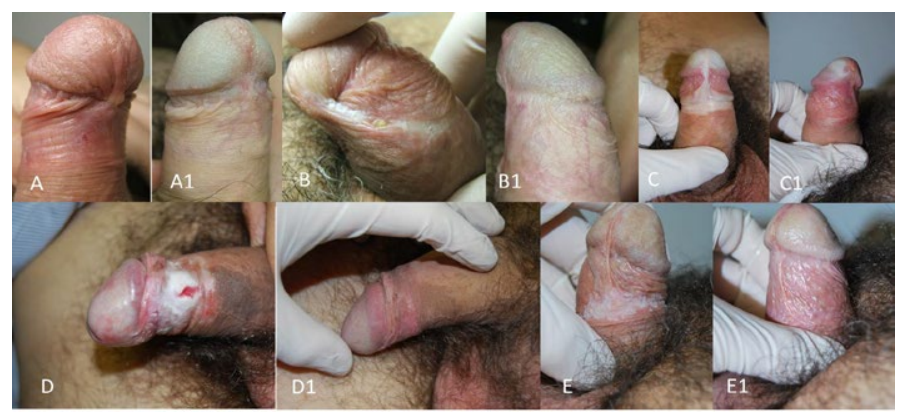

Figure 1. Notice, the significant improvement of Lichen sclerosus signs after combined therapy (ex. A: pre-therapy; A1: post-therapy).

immediately intractable with only topical, systemic or micro infiltrative approaches, due to the mentioned alterations. In this context it could be very useful, to compose a multi-dimensional approach, which must include in order:

1. A conservative dermo-surgical approach, always associated by histological examination (even on dermatologic LS lesions deemed not treatable with medical therapy), which range in men, by the removal of leukoplakia or suspicious lesions of gland or prepuce; frenuloplasty; partial or total circumcision; in women: minor or major labia excision of suspicious lesions and vulvo-vaginal plastic due to a reduction of vulvo-vaginal orifice.

\section{Topical therapy}

3. Micro-infiltrative Therapy (rich-platelet-plasma, polydeoxyribonucleotide)

In our opinion, we sustain how this path should not be reversed, according to a common sense rule that should first:

a) Remove the anatomical functional impediments which potentially could delay or prohibits the functioning of medical therapies

b) Obtain the histological evaluation before any topical (ex. steroid, topical immunomodulators) application, in order to have a clear reading of histological preparations

c) Immediately exclude the existence of pre-neoplastic or neoplastic cell changes, which may require additional surgical approaches and consecutively shut out other therapies (ex. topical steroid/immunomodulators and/or micro-infiltrative approaches)

In our opinion, only after this approach, in the mentioned cases, is useful to proceed, with topical and/or micro-infiltrative therapy, that can't constitute a unique medical approach, but only an adjuvant therapy.
The choice of topical therapy should, always fall back on a medium to high potential steroidal drug, or topical immunomodulators according to the last published guidelines about LS. Is also useful, to conduct a micro-infiltrative therapy, with PRP or with Polydeoxyribonucleotide, in order to contribute in tissue regenerative approach already affected by the disease. We use in all selected cases, Polydeoxyribonucloeotide (PDRN) subdermal-infiltrations, following the guidelines of our protocol $[8,9]$. Thanks to this therapeutic triad, we have achieved in all our patients, significant $(\mathrm{p}<0.001)$ decrease of IGA and DLQI (Table 1) even in case of severe LS; these clinical results (Figure 1), were maintained at a distance of months from the suspension of the therapy.

Despite many therapeutic approaches proposed over the years, the definitive treatment of lichen sclerosus is still being codified. We believe that a viable therapy consists of a multidisciplinary approach that is based on proper clinical staging and histological diagnosis. We maintain that a valid therapeutic proposal is not confined to a drug, but to a therapeutic set and to a proper therapeutic approach, based on the type and the severity degree of the disease. In this context, through this preliminary study, we have highlighted the efficacy, tolerability, and safety profile demonstrated by a therapeutic dermatologic triad, which could be cited, (if further studies confirm these early data) as one of the effective management of lichen sclerosus.

\section{References}

1. Cavelier-Balloy B (2012) Lichen sclerosus. Ann Dermatol Venereol 139: 65-67. [Crossref]

2. Lü J, Huang XD (2014) Current diagnosis and treatment of male genital lichen sclerosus. Zhonghua Nan Ke Xue 20: 579-585. [Crossref]

3. Fistarol SK, Itin PH (2013) Diagnosis and treatment of lichen sclerosus: an update. $\mathrm{Am}$ J Clin Dermatol 14: 27-47. [Crossref]

4. Brady KL, Mercurio MG, Brown MD (2013) Malignant tumors of the penis. Dermatol Surg 39: 527-547. [Crossref]

5. Neill SM, Lewis FM, Tatnall FM, Cox NH (2010) British association of dermatologists' guidelines for the management of lichen sclerosus 2010. Br J Dermatol 163: 672-682. [Crossref]

6. Finlay AY, Khan GK (1994) Dermatology Life Quality Index (DLQI) — a simple practical measure for routine clinical use. Clin Exp Dermatol 19: 210-216. [Crossref]

7. Bitto A, Polito F, Irrera N, D'Ascola A, Avenoso A, et al. (2011) Polydeoxyribonucleotide reduces cytokine production and the severity of collagen-induced arthritis by stimulation of adenosine A2A receptor. Arthritis Rheum 63: 3364-3371. [Crossref]

8. Laino (2012) Adjuvant clinical effects of polydeoxyribonucleotide in lichen sclerosus. Eur J Dermatol 22: 575-576. [Crossref]

9. Laino L, Suetti S, Sperduti I (2013) Polydeoxyribonucleotide Dermal Infiltration in Male Genital Lichen Sclerosus: Adjuvant Effects during Topical Therapy. Dermatol Res Pract 2013: 654079.

Copyright: (C2015 Laino L. This is an open-access article distributed under the terms of the Creative Commons Attribution License, which permits unrestricted use, distribution, and reproduction in any medium, provided the original author and source are credited. 